\title{
PROPERTIES OF PARTIALLY EXCITED SIBERIAN SNAKES
}

\author{
Thomas Roser \\ Randall Laboratory of Physics, The University of Michigan \\ Ann Arbor, MI 48109
}

\section{ABSTRACT}

The properties of the insertion of a general spin rotator into a circular accelerator are examined. It is shown that even for a small rotation angle depolarization from weak imperfection resonances is avoided.

\section{INTRODUCTION}

It is well known that a Siberian Snake - a device that rotates the spin by $180^{\circ}$ around a horizontal direction - is expected to eliminate the effects of strong depolarization resonances that occur in circular accelerators. It is thus natural to ask the question whether a partially excited Siberian Snake - a device that rotates the spin by $\delta<180^{\circ}$ around a horizontal direction would eliminate the effect of weak depolarizing resonances as they occur in low energy machines ( $<50 \mathrm{GeV}$ ). Such a partially excited Siberian Snake would have the additional advantage of reducing the orbit excursions that occur in the standard configuration of a Siberian Snake of a sequence of vertical and horizontal bends.

\section{SPIN TUNE}

It is easy to show that the insertion of a fully excited Siberian Snake in a accelerator causes the spin tune $\nu_{s}$ to become $1 / 2$. As a result none of the conditions for depolarizing resonances

$$
\begin{array}{ccc}
\nu_{s}=n & (\text { imperfection) } & n: \text { integer } \\
\nu_{a}=n P \pm \nu_{y} & \text { (intrinsic) } & P: \text { superperiodicity }
\end{array}
$$

can be satisfied provided the vertical betatron tune $\nu_{y}$ is not a half-integer.

The spin tune in the presence of a partially excited Siberian Snake can be extracted from the one-turn spin transport matrix $M$ (Ref 1.). $M$ is obtained as a product of a spin rotation in the Siberian Snake by an angle $\delta$ around the longitudinal direction and the precession in the main bending field by the angle $2 \pi G \gamma$ around the vertical direction ( $G$ : anomalous magnetic moment, $\gamma=E / m)$ :

$$
M=\left[\cos (\pi G \gamma)-i \sigma_{y} \sin (\pi G \gamma)\right]\left[\cos \left(\frac{\delta}{2}\right)-i \sigma_{z} \sin \left(\frac{\delta}{2}\right)\right]
$$

The spin tune is then obtained from the trace of $\mathrm{M}$ :

$$
\cos \left(\pi \nu_{s}\right)=\frac{1}{2} \operatorname{Tr}(M)=\cos (\pi G \gamma) \cos \left(\frac{\delta}{2}\right)
$$




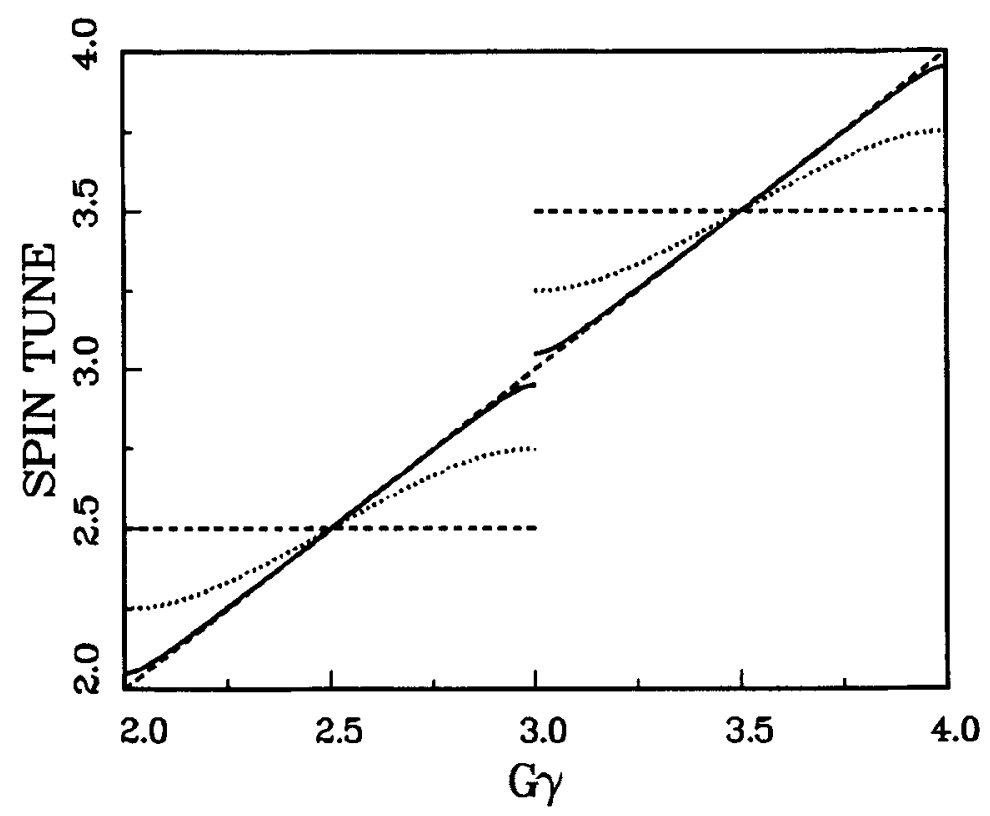

Figure 1.Spin tune for $\delta=0^{\circ}$ (dashed diagonal line), $\delta=18^{\circ}$ (solid), $\delta=90^{\circ}$ (dotted) and $\delta=180^{\circ}$ (dashed horizontal lines).

Notice that this equation has the correct limits:

$$
\begin{array}{cll}
\text { full Snake }: & \delta=\pi \rightarrow \nu_{s}=\frac{1}{2} \\
\text { no Snake }: & \delta=0 \rightarrow \nu_{s}=G \gamma
\end{array}
$$

Fig. 1 shows a graph of Eq. (3) for various values of $\delta$. The graph clearly shows that even for small values of $\delta$ the spin tune never assumes integer values. In fact the 'forbidden region' for the spin tune around the integers widens with increasing angle $\delta$ according to the relation:

$$
\Delta=\frac{\delta}{\pi}
$$

reaching one for a full Snake. Intuitively the size of this 'forbidden region' indicates the maximum strength of a depolarizing resonance that can be eliminated. 
DEPOLARIZATION FROM A PARTIALLY EXCITED SIBERIAN SNAKE

Froissart and Stora ${ }^{2}$ obtained an analytic solution for the amount of depolarization in the passage through a single isolated resonance with strength $\epsilon$ :

$$
\frac{P_{\text {final }}}{P_{\text {initial }}}=2 \exp \left(-\frac{\epsilon^{2} \pi}{2 \alpha}\right)-1
$$

Similarly the depolarization from the passage through an integer resonance generated by a partially excited Siberian Snake can be estimated. We can write the localized spin rotation from the Siberian Snake at the turning angle $\theta_{0}$ as a delta-function:

$$
\delta \times \delta\left(\theta-\theta_{0}\right)
$$

The strength of the generated depolarizing resonance at integer $n_{0}$ is simply the amplitude of the corresponding Fourier component:

$$
\frac{\delta}{2 \pi} \exp \left(i n_{0} \theta_{0}\right)
$$

The formula for depolarization is then

$$
\frac{P_{\text {final }}}{P_{\text {initial }}}=2 \exp \left(-\frac{\delta^{2}}{8 \pi \alpha}\right)-1
$$

Notice that for

$$
\delta \gg \sqrt{8 \pi \alpha}
$$

a partially excited Siberian Snake generates complete spin-flip at every integer resonance. For the AGS $\sqrt{8 \pi \alpha}$ is only $\sim 2^{\circ}$. At smaller values, however, the Snake will cause depolarization!

In the presence of an imperfection resonance with complex strength $\epsilon$ Eq. (9) becomes

$$
\frac{P_{\text {final }}}{P_{\text {initial }}}=2 \exp \left(-\frac{\pi}{2 \alpha}\left|\epsilon+\frac{\delta}{2 \pi} \exp \left(i n_{0} \theta_{0}\right)\right|^{2}\right)-1
$$

In this case complete spin-flip occurs if

$$
\delta \gg 2 \pi|\epsilon|+\sqrt{8 \pi \alpha}
$$

For the AGS with $|\epsilon|<10^{-2}$ this means $\delta \gg 4^{\circ}$ ensures complete spin-flip at every integer resonance.

Fig. 2 shows the result of a simulation calculation for a partially excited Siberian Snake with $\delta=9^{\circ}$. The spin was tracked through a integer resonance and the final polarization was plotted as a function of the strength of the 


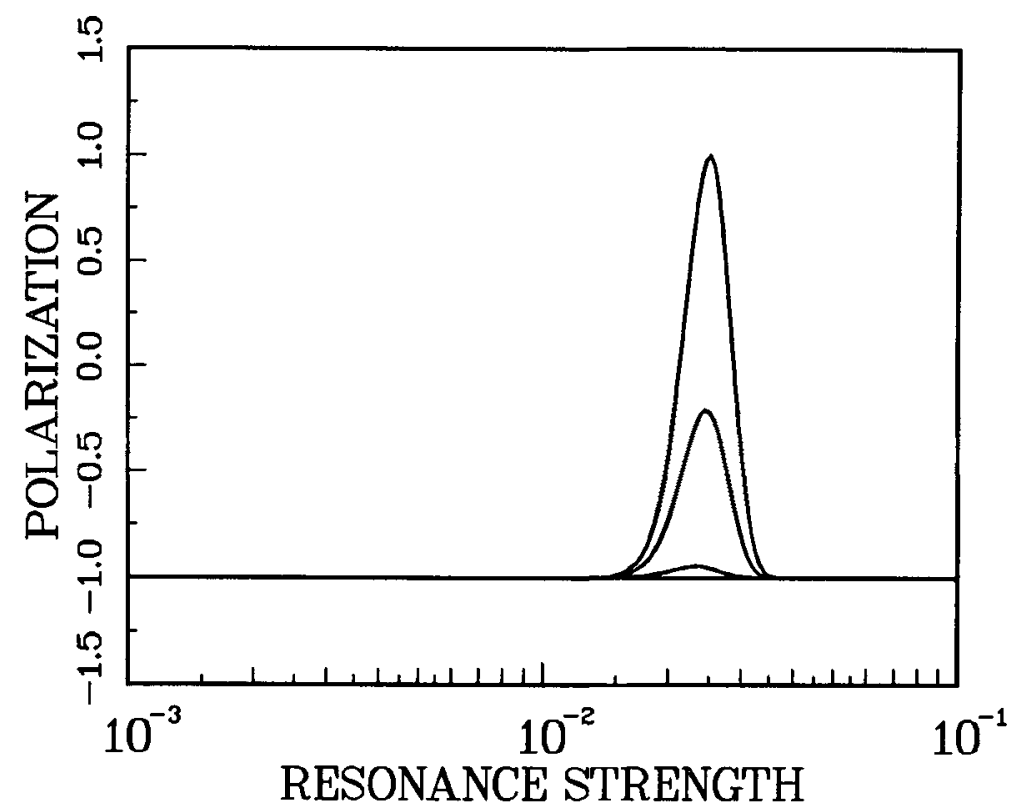

Figure 2.Tracking result with $\delta=9^{\circ}$ partially excited Siberian Snake for various values of $\theta_{0}$.

depolarizing resonance. The different curves correspond to different values of $\theta_{0}$. Clearly a 9 degree Siberian Snake eliminates depolarization in the AGS.

\section{POLARIZATION DIRECTION}

With a single partially excited Siberian Snake inserted in an accelerator the stable polarization direction will no longer be vertical but tilted away from the vertical direction by an amount the depends both on energy and turning angle. The stable polarization direction can easily be determined from the one-turn matrix M:

$$
\left(P_{x}, P_{y}, P_{z}\right)=\left(\sin [(\pi-\theta) G \gamma] \sin \frac{\delta}{2}, \sin \pi G \gamma \cos \frac{\delta}{2}, \cos [(\pi-\theta) G \gamma] \sin \frac{\delta}{2}\right)
$$

Fig. 3 shows the vertical polarization for $\delta=9^{\circ}$ indicating that between integer values of $G \gamma$ the beam is almost fully vertically polarized.

\section{CONCLUSIONS}

A partially excited Siberian Snake offers an interesting possibility to eliminate the effect of weak imperfection resonances as they occur in low energy accelerators. It can be realized with minimal space requirements and minimal induced orbit excursions 


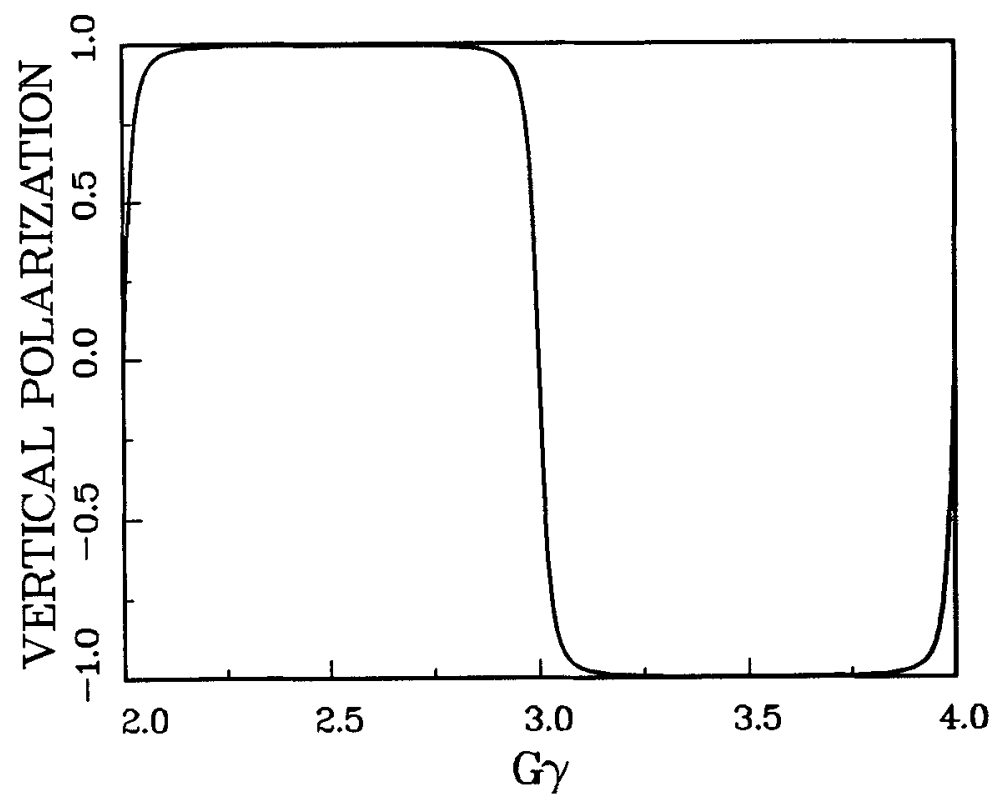

Figure 3.Vertical polarization for a $\delta=9^{\circ}$ partially excited Siberian Snake.

This work was supported by a grant from the U.S. Department of Energy.

\section{REFERENCES}

1. S.Y.Lee, S.Tepikian and E.Courant in Polarized Beams at the SSC, AIP Conference Proceedings 145 (1986) 185

2. M.Froissart and R.Stora, Nucl. Inst. and Methods 1 (1960) 297 